\title{
A new model to study the phase transition from microstructures to nanostructures in ionic/ionic surfactants mixture
}

\section{Beheshteh Sohrabi, ${ }^{*}{ }^{\dagger},{ }^{\ddagger}$ Hussein Gharibi, ${ }^{\dagger}{ }^{\S}$ Soheila Javadian, ${ }^{\dagger}$ and Majid Hashemianzadeh"}

${ }^{\dagger}$ Department of Chemistry, Tarbiat Modarres University, P.O.Box 14155-4838, Tehran, Iran

${ }^{\ddagger}$ Peresent Address:Department of Chemistry, Shahid Rajaee Teacher Traing University, P.O.Box 16785-163, Tehran, Iran

Iranian Information and Documentation Center, IRANDOC, P.O. Box 13185-1371, Tehran, Iran

"Department of Chemistry, College of Chemistry, Iran University of Science and Technology, P.O. Box 16765-163, Tehran, Iran

Supporting information 


\section{A. Determination of surface potentials in vesicles by the capacitor model}

To determine the surface potential, we considered each of the two vesicle layers as similar to an electrical double layer or capacitor. Since a vesicle possesses four surface potentials and one electrical potential in hydrophobic region since vesicle surfaces possess ions and water molecules and the tail of surfactants rests in hydrophobic region of vesicle and assumed the ions can freely cross but not accumulate in this region. The potential difference of each capacitor must be taken into account. The surface potentials can be calculated by solving the PB equation according to:

$\frac{d^{2} y}{d x^{2}}+\frac{2}{x} \frac{d y}{d x}=\sinh y$

Where

$y_{j}=\frac{e z \psi_{j}}{k T}, \quad j=1-4$

$x=\kappa_{D} r$

$\kappa_{D}=\sqrt{\frac{4 \pi n_{0} e^{2} z^{2}}{\varepsilon_{w} k T}}$

Here, $\psi_{j}$ is the electrostatic potential in region $j, y_{j}$ is the reduced potential in region $j, r$ is the radial coordinate, $\kappa_{D}$ is the inverse of the Debye screening length, $\varepsilon_{w}=4 \pi \eta_{w} \varepsilon_{0}$ is the permittivity of water (where $\eta_{w}$ is the dielectric constant of water, and $\varepsilon_{0}$ is the permittivity of vacuum), $k$ is Boltzmann's constant, $e$ is the elementary charge, $T$ is the absolute temperature, and $n_{0}=C N_{A} / 1000$ (where $C$ is the total concentration of

surfactants). ${ }^{1}$ The work required to arrange charge on the innermost layer 
gives the electrical potential of the first layer. The nonlinear PB equation for this region can be written as follows:

$$
\int_{0}^{y_{1}^{\prime}} \frac{d}{d y}\left(\frac{d y}{d x}\right)^{2} d y=2 \int_{0}^{y_{1}^{\prime}} \sinh y d y-\frac{4}{x} \int_{0}^{y_{1}^{\prime}} \frac{d y}{d x} d y
$$

Integral limits are taken from the center to the first layer of the vesicle.

The planar approximation has been used to calculate the second integral on the right side. ${ }^{1}$

$-\frac{d y}{d x}=2 \sinh \frac{y_{0}}{2}$

Therefore

$$
\left.\left(\frac{d y}{d x}\right)^{2}\right|_{x=x_{i}^{\prime}}=\left.2 \cosh y\right|_{0} ^{y_{1}^{\prime}}-\frac{4}{x_{i}^{\prime}} \int_{0}^{y_{1}^{\prime}} 2 \sinh \frac{y}{2} d y
$$

The electrical potential in the vesicle center is negligible..$^{2-5}$ Thus, taking $y_{0}=0$, we obtain:

$$
\begin{aligned}
\left.\left(\frac{d y}{d x}\right)^{2}\right|_{x=x_{i}^{\prime}} & =2\left(\cosh y_{1}^{\prime}-1\right)-\left.\frac{16}{x_{i}^{\prime}} \cosh \frac{y}{2}\right|_{0} ^{y_{1}^{\prime}} \\
& =4 \sinh ^{2} \frac{y_{1}^{\prime}}{2}-\frac{16}{x_{i}^{\prime}}\left[\cosh \frac{y_{1}^{\prime}}{2}-1\right]
\end{aligned}
$$


$\left.\left(\frac{d y}{d x}\right)^{2}\right|_{x=x_{i}^{\prime}}=4 \sinh ^{2} \frac{y_{1}^{\prime}}{2}\left(1-\frac{4}{x_{i}^{\prime}} \frac{\left(\cosh \frac{y_{1}^{\prime}}{2}-1\right)}{\sinh ^{2} \frac{y_{1}^{\prime}}{2}}\right)^{1 / 2}$
$\left.\frac{d y}{d x}\right|_{x=x_{i}^{\prime}}=2 \sinh \frac{y_{1}^{\prime}}{2}\left(1-\frac{4}{x_{i}^{\prime}} \frac{1}{\left(\cosh \frac{y_{1}^{\prime}}{2}+1\right)}\right)^{1 / 2}$

To calculate the electrical potential of the second innermost layer of the vesicle, the innermost layer must be uncharged. Since the works of charging and discharging the innermost layer differ only in sign, the solution of the nonlinear PB equation for the second innermost layer gives:

$$
\left.\frac{d y}{d x}\right|_{x=x_{i}}=2 \sinh \frac{y_{1}}{2}\left(1-\frac{4}{x_{i}} \frac{1}{\left(\cosh \frac{y_{1}}{2}+1\right)}\right)^{1 / 2}
$$

For the outer capacitor, the solution is:

$$
\begin{aligned}
& \left.\frac{d y}{d x}\right|_{x=x_{o}}=-2 \sinh \frac{y_{3}}{2}\left(1+\frac{4}{x_{o}} \frac{1}{\left(\cosh \frac{y_{3}}{2}+1\right)}\right)^{1 / 2} \\
& \left.\frac{d y}{d x}\right|_{x=x_{o}^{\prime}}=-2 \sinh \frac{y_{3}^{\prime}}{2}\left(1+\frac{4}{x_{o}^{\prime}} \frac{1}{\left(\cosh \frac{y_{3}^{\prime}}{2}+1\right)}\right)^{1 / 2}
\end{aligned}
$$

And in the hydrophobic region,

$$
\frac{d^{2} y}{d x^{2}}+\frac{2}{x} \frac{d y}{d x}=0
$$

Because the charge density in the hydrophobic region is zero, the PB equation is equal to zero. This homogeneous differential equation can be 
solved exactly to give the potential gradients at the two surfaces, specifically,

$$
\begin{aligned}
& \left.\frac{d y_{2}}{d x}\right|_{x=x_{i}}=\frac{1}{x_{i}^{2}}\left(\frac{1}{x_{i}}-\frac{1}{x_{o}}\right)^{-1}\left(y_{3}-y_{1}\right) \\
& \left.\frac{d y_{2}}{d x}\right|_{x=x_{o}}=\frac{1}{x_{o}^{2}}\left(\frac{1}{x_{i}}-\frac{1}{x_{o}}\right)^{-1}\left(y_{3}-y_{1}\right)
\end{aligned}
$$

The boundary conditions for the set of differential eqs 18 - 22 can be written as follows:

a) At $x=0$

$$
\begin{aligned}
& \left.\frac{d y}{d x}\right|_{x=x_{i}^{\prime}}=0 \\
& y_{1}=y_{0}
\end{aligned}
$$

b) At $x=\kappa_{D} R_{i} \equiv X_{i}$

$\left.\varepsilon_{1} \kappa_{D} \frac{d y}{d x}\right|_{x=X_{i}}-\left.\varepsilon_{2} \kappa_{D} \frac{d y_{2}}{d x}\right|_{x=X_{i}}=\frac{4 \pi \sigma_{1} e z}{k T}$

$$
y_{1}=y_{2}
$$

c) At $x=\kappa_{D} R_{o} \equiv X_{o}$ 
$\left.\mathcal{E}_{2} \kappa_{D} \frac{d y_{2}}{d x}\right|_{x=X_{o}}-\left.\varepsilon_{3} \kappa_{D} \frac{d y}{d x}\right|_{x=X_{o}}=\frac{4 \pi \sigma_{3} e z}{k T}$

$y_{2}=y_{3}$

d) As $x \rightarrow \infty$

$\left.\frac{d y}{d x}\right|_{x=x_{o}^{\prime}}=0$

$y_{3}^{\prime}=0$

Where $\sigma_{l}$ and $\sigma_{3}$ are the charge densities at the second and third surfaces, respectively.

For simplicity, we assume that the system is comprised of only two regions with distinct dielectric constant, namely water and the hydrophobic region and that other effects such as dielectric saturation can be neglected. ${ }^{24}$ Although regions 1 and 3 containing ionic solutions will have lower dielectric constants than pure water, ${ }^{24} \varepsilon_{1}$ and $\varepsilon_{3}$ have been assumed to equal $\varepsilon_{w}$.

The dielectric constant is set to 2.5 for the hydrophobic region and 78.54 for water, ${ }^{5}$ and all the calculations are carried out at $25^{\circ} \mathrm{C}$. Substituting eqs 18 , 20, 23 and 24 into eqs 27 and 29, we obtain the following two equations:

$$
\begin{gathered}
\varepsilon_{1} \kappa_{D} 2 \sinh \frac{y_{1}}{2}\left(1-\frac{4}{X_{i}} \frac{1}{\left(\cosh \frac{y_{1}}{2}+1\right)}\right)^{1 / 2}-\varepsilon_{2} \kappa_{D} \frac{1}{X_{i}^{2}}\left(\frac{1}{X_{i}}-\frac{1}{X_{o}}\right)^{-1}\left(y_{3}-y_{1}\right)=\frac{4 \pi \sigma_{1} z e}{k T} \\
\varepsilon_{2} \kappa_{D} \frac{1}{X_{o}^{2}}\left(\frac{1}{X_{i}}-\frac{1}{X_{o}}\right)^{-1}\left(y_{3}-y_{1}\right)+\varepsilon_{3} \kappa_{D} 2 \sinh \frac{y_{3}}{2}\left(1+\frac{4}{X_{o}} \frac{1}{\left(\cosh \frac{y_{3}}{2}+1\right)}\right)^{1 / 2}=\frac{4 \pi \sigma_{3} z e}{k T}
\end{gathered}
$$

It can be considered that the pair of interior layers and the pair of outer layers each act as capacitors. Therefore, for each of these pairs of layers, the potential difference between the layers can be determined by Gauss's law: 
If there is a dielectric medium between the conductors, the medium will be polarized and the polarization charge will be opposite in sign to the induced charge. The effect of the applied charge $q$ is reduced by a factor $\eta_{\mathrm{r}}$ and the potential between the conductors is reduced by the same factor. Consider an isolated sphere of radius $a$ and charge $q$ in an infinite dielectric medium of dielectric constant $\eta_{\mathrm{r}}$. We apply Gauss's theorem over a spherical surface of radius $r$, concentric with the isolated sphere:

$$
\int D_{s} \cdot d s=q
$$

$D_{s}$ is constant over the spherical surface; thus by integrating we obtain

$$
\begin{aligned}
& 4 \pi r^{2} D_{s}=q=4 \pi \eta_{w} \varepsilon_{0} r^{2} E \\
& E=\frac{q}{4 \pi \eta_{w} \varepsilon_{0} r^{2}}
\end{aligned}
$$

The potential of the sphere $(r=a)$ is

$$
V=-\int_{\infty}^{a} E d r=\frac{q}{4 \pi \eta_{w} \varepsilon_{0} a}
$$

With two concentric spheres (e.g., vesicles) of radius a and b and charge $q$ and $-\mathrm{q}^{\prime}$ respectively, the field at a Gaussian sphere of radius $\mathrm{r}$ (where $\mathrm{a}<\mathrm{r}<$ b) is given by eq A.30. When $r$ is outside the larger sphere the field at $r$ is zero since the total charge enclosed by any surface larger than the outer sphere is zero.

The potential between the two spheres is therefore

$$
V=-\int_{a}^{b} E d r=\frac{q}{4 \pi \eta_{w} \varepsilon_{0}}\left(\frac{1}{a}-\frac{1}{b}\right)
$$

For vesicles, A.32 takes on the form: 


$$
\begin{aligned}
& y_{1}^{\prime}-y_{1}=\frac{Q_{1}^{\prime} e \kappa_{D}}{4 \pi \varepsilon_{0} \eta_{w} k T}\left(\frac{1}{X_{i}^{\prime}}-\frac{1}{X_{i}}\right) \\
& y_{3}-y_{3}^{\prime}=\frac{Q_{3} e \kappa_{D}}{4 \pi \varepsilon_{0} \eta_{w} k T}\left(\frac{1}{X_{o}}-\frac{1}{X_{o}^{\prime}}\right)
\end{aligned}
$$

Where $y_{1}, y_{1}^{\prime}, y_{3}$ and $y_{3}^{\prime}$ are the reduced surface potentials on each of the capacitor layers, $\eta_{w}$ is the dielectric constant of water, and $X_{\mathrm{i}}^{\prime}, X_{\mathrm{i}}, X_{\mathrm{o}}$ and $X_{\mathrm{o}}^{\prime}$ are the reduced radii of each of the capacitor layers.

The surface potentials were calculated by simultaneous solution of eqs A.26 - A.34 using the software Mathematica. ${ }^{6}$

\section{B. Determination of the surface potentials in vesicle}

According to Figure 1A, the surface potential is negative for surfaces formed from SDS. In addition, the inner surface potential exceeds the outer surface potential, indicating that the inner charge density is greater than the outer charge density. In the cationic-rich region, it is very likely that the CTAB molecules initially form micelles, and then the SDS molecules penetrate into the CTAB micelles to form mixed micelles. One effect of attractive force between the CTAB and SDS monomers is to increase the amount of SDS monomers on the outer vesicle layer. Since the opposite charges almost cancel each other; consequently, the inner surface potential will be larger than the outer surface potential. Figure $2 \mathrm{~A}$ shows the variation of surface electrical potential as a function of SDS concentration in the anionic-rich regime. It is clear from this plot that the SDS molecules are located in the first and fourth layers. 
[1] Bockris, J. O.; Reddy, A. K. N. Modern electrochemistry, Vol.1, Chap.3, Plenum Press, New York and London, 1998.

[2] Evans, D. F.; Mitchell, D. J.; Ninham, B. W. J. phys. chem. 1984, 88, 6344.

[3] Evans, D. F.; Ninham, B. W. J. phys. chem. 1983, 87, 5025.

[4] Mitchell, D. J.; Ninham, B. W. J. phys. chem. 1983, 87, 2996.

[5] Yuet, P. K.; Blankschtein, D. Langmuir. 1995, 11, 1925.

[6] Wolfram, S. Mathematica, Addison-Wesley, 1988. Kreyzig, E.; Normiton, E. J. Mathematica Computer Guide, John Wiley \& Sons: New York, 2002. http://www.wolram.com.

Table A. Data for vesicles in cataionic mixtures with different total concentrations and constant mole fraction (CTAB/SDS, 0.66/0.34).

Figure 1A. Surface potential in different vesicle layers vs. CTAB concentration at a constant mole fraction of $0.66 / 0.34$ (CTAB/SDS).

Figure 2A. Variation of surface potential in different vesicle layers vs. SDS concentration at a constant mole fraction of $0.25 / 0.75$ (CTAB/SDS). 
Table 2A

\begin{tabular}{|c|c|c|c|c|c|c|c|c|c|c|c|c|}
\hline $\mathrm{C}_{\mathrm{tot}}(\mathrm{mM})$ & $\mathrm{N}_{\mathrm{tot}} \times 10^{-6}$ & $\mathrm{f}$ & $\mathrm{X}_{\mathrm{CTAB}, \mathrm{i}}$ & $\mathrm{X}_{\mathrm{CTAB}, \mathrm{o}}$ & $\mathrm{X}_{\mathrm{SDS}, \mathrm{i}}$ & $\mathrm{X}_{\mathrm{SDS}, \mathrm{o}}$ & $\mathrm{N}_{\mathrm{CTAB}, \mathrm{i}} \times 10^{-6}$ & $\mathrm{~N}_{\mathrm{CTAB}, \mathrm{o}} \times 10^{-6}$ & $\mathrm{~N}_{\mathrm{SDS}, \mathrm{o}} \times 10^{-5}$ & $\mathrm{~N}_{\mathrm{SDS}, \mathrm{o}} \times 10^{-5}$ & $\mathrm{Q}_{\mathrm{i}} \times 10^{13}$ & $\mathrm{Q}_{\mathrm{o}} \times 10^{13}$ \\
\hline 0.730 & 5.47 & 0.510 & 0.6873 & 0.6836 & 0.3127 & 0.3164 & 1.84 & 1.91 & 8.39 & 8.82 & 1.61 & 1.64 \\
\hline 0.663 & 5.57 & 0.509 & 0.6886 & 0.6844 & 0.3114 & 0.3156 & 1.88 & 1.94 & 8.51 & 8.96 & 1.65 & 1.68 \\
\hline 0.608 & 5.59 & 0.509 & 0.6887 & 0.6843 & 0.3113 & 0.3157 & 1.89 & 1.95 & 8.53 & 8.98 & 1.66 & 1.68 \\
\hline 0.561 & 5.32 & 0.510 & 0.6873 & 0.6836 & 0.3127 & 0.3164 & 1.79 & 1.86 & 8.16 & 8.59 & 1.57 & 1.60 \\
\hline 0.522 & 5.69 & 0.509 & 0.6872 & 0.6836 & 0.3128 & 0.3164 & 1.92 & 1.98 & 8.73 & 9.17 & 1.67 & 1.70 \\
\hline 0.487 & 5.77 & 0.509 & 0.6872 & 0.6836 & 0.3128 & 0.3164 & 1.95 & 2.01 & 8.86 & 9.30 & 1.70 & 1.73 \\
\hline 0.456 & 5.89 & 0.509 & 0.6871 & 0.6836 & 0.3129 & 0.3164 & 1.99 & 2.05 & 9.04 & 9.49 & 1.73 & 1.76 \\
\hline 0.417 & 5.82 & 0.509 & 0.6872 & 0.6836 & 0.3128 & 0.3164 & 1.96 & 2.03 & 8.94 & 9.39 & 1.71 & 1.75 \\
\hline 0.394 & 5.54 & 0.510 & 0.6872 & 0.6836 & 0.3128 & 0.3164 & 1.87 & 1.93 & 8.49 & 8.92 & 1.63 & 1.66 \\
\hline 0.365 & 5.86 & 0.509 & 0.6872 & 0.6836 & 0.3128 & 0.3164 & 1.98 & 2.04 & 9.00 & 9.44 & 1.72 & 1.76 \\
\hline 0.339 & 6.05 & 0.509 & 0.6871 & 0.6836 & 0.3120 & 0.3164 & 2.04 & 2.11 & 9.30 & 9.75 & 1.78 & 1.81 \\
\hline 0.324 & 5.39 & 0.510 & 0.6873 & 0.6836 & 0.3127 & 0.3164 & 1.82 & 1.88 & 8.27 & 8.70 & 1.59 & 1.62 \\
\hline
\end{tabular}




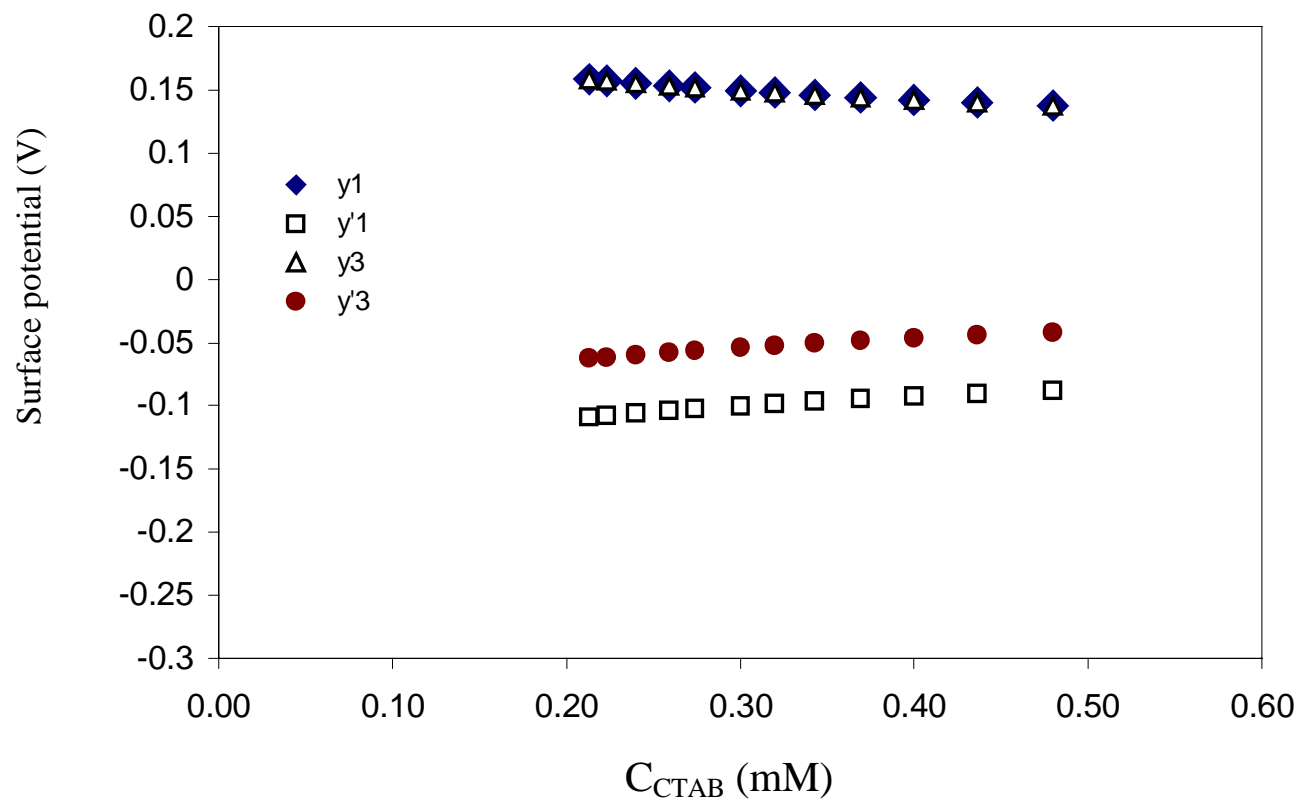

Figure 1A 


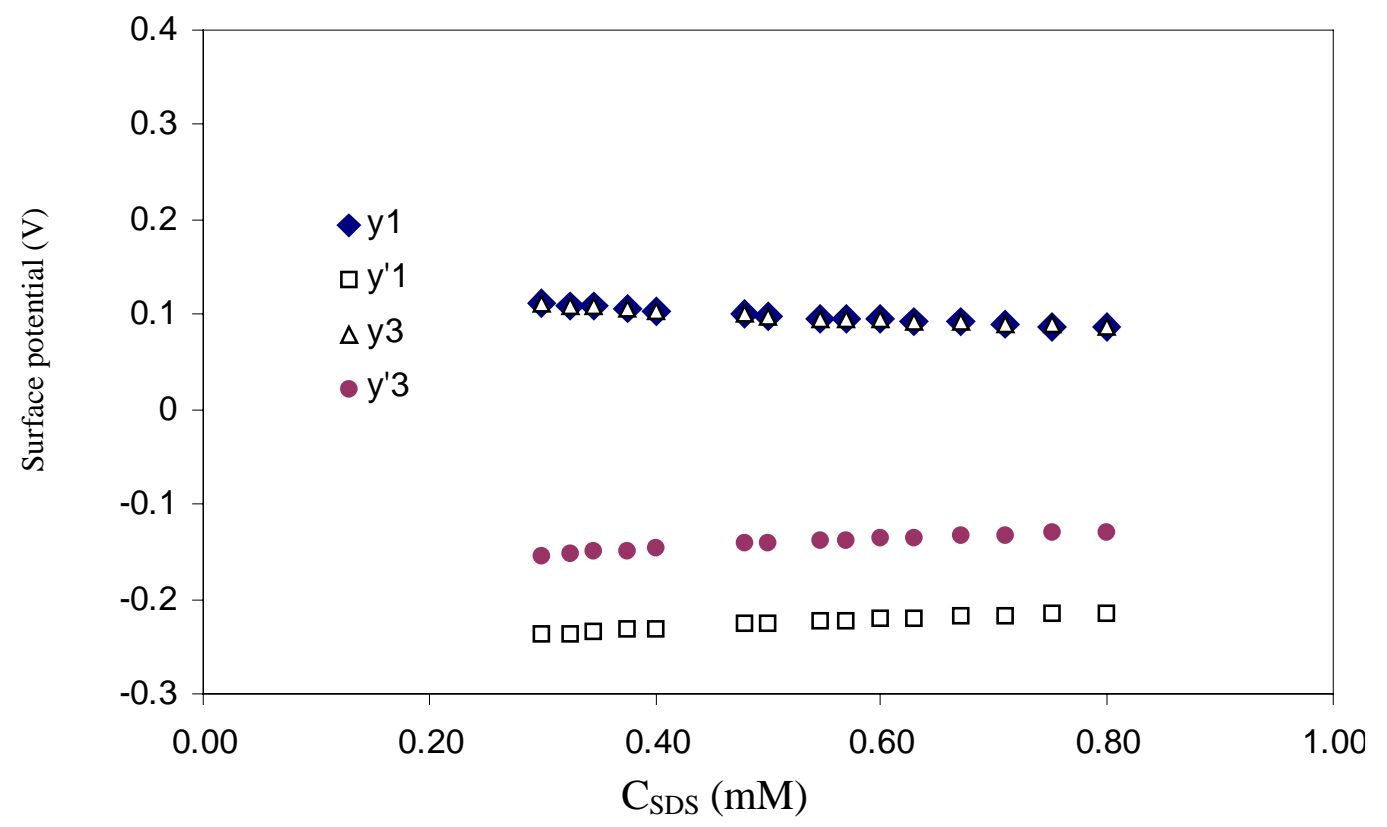

Figure 2A 